\title{
La emergencia de las «empresas de base humana» en España: ¿nuevo paradigma o consecuencia inevitable?
}

\author{
Hugo Valenzuela García \\ José Luis Molina \\ Universidad Autónoma de Barcelona. Departamento de Antropología Social y Cultural \\ hugo.valenzuela@uab.es \\ joseluis.molina@uab.es
}

Recepción: septiembre de 2012

Aceptación: marzo de 2013

\begin{abstract}
Resumen
El artículo presenta una serie de estudios de caso etnográfico de empresas españolas de "base humana», denominadas de este modo por potenciar el factor humano como ventaja competitiva. Estas empresas poseen ciertos rasgos en común: operan en la economía del conocimiento, sus fundadores son talentosos jóvenes emprendedores, aplican conocimientos de las humanidades, son flexibles y cooperativas, realizan una gestión horizontal y no persiguen el lucro como único objetivo. Tales empresas despuntan en oposición a los modelos de gestión y producción empresarial e industrial tradicionales y ponen en primer plano la innovación centrada en las personas frente a la innovación tecnoeconómica clásica. Sin embargo, ¿`son realmente pioneras?, ¿suponen un cambio de paradigma en la gestión y organización empresarial en nuestro país?, ¿o acaso son una consecuencia lógica de la producción flexible, la mercantilización expansiva del capitalismo tardío y un eco de iniciativas que irradian de otros centros neurálgicos empresariales?
\end{abstract}

Palabras clave: innovación; etnografía corporativa; alienación; postfordismo; tangibles; empresas de base humana; factor humano; gestión de recursos humanos; creación/destrucción de empresas.

Resum. L'emergència de les "empreses de base humana» a Espanya: nou paradigma o conseqüència inevitable?

L'article exposa una sèrie d'estudis de cas etnogràfic d'empreses espanyoles de «base humana", anomenades d'aquesta manera pel fet de potenciar el factor humà com a avantatge competitiu. Aquestes empreses posseeixen certs trets en comú: operen en l'economia del coneixement, els seus fundadors són joves emprenedors talentosos, apliquen coneixements de les humanitats, són flexibles i cooperatives, tenen una gestió horitzontal i no perseguei- 
xen el lucre com a objectiu únic. Aquestes empreses despunten en oposició als models de gestió i producció industrial i de negocis tradicionals, i posen en primer pla la innovació centrada en les persones davant la innovació tecnològica econòmica clàssica. Ara bé, són realment pioneres?, suposen un canvi de paradigma en la gestió i organització empresarial a casa nostra?, o potser són una conseqüència lògica de la producció flexible, la mercantilització expansiva del capitalisme tardà i un ressò d'iniciatives que irradien d'altres centres neuràlgics empresarials?

Paraules clau: innovació; etnografia corporativa; alienació; postfordisme; intangibles; empreses de base humana; factor humà; gestió de recursos humans; creació/destrucció d'empreses.

Résumé. L'émergence des «sociétés d'origine humaine» en Espagne : nouveau paradigme ou un conséquence inévitable?

L'article présente un ensemble d'études de cas ethnographiques portant sur des entreprises espagnoles «de base humaine», dénommées ainsi afin de favoriser le facteur humain comme avantage concurrentiel. Ces entreprises ont certains éléments en commun: elles opèrent sur l'économie de la connaissance, leurs fondateurs sont de jeunes entrepreneurs talentueux, appliquent des connaissances dans le domaine des humanités, sont flexibles et coopératives, pratiquent une gestion horizontale et leur objectif n'est pas uniquement l'appât du gain. De telles entreprises s'opposent aux modèles de gestion et de production d'entreprises industrielles traditionnelles et mettent au premier plan l'innovation centrée sur les personnes plutôt que l'innovation techno-économique classique. Cependant, ces entreprises sont-elle réellement pionnières ? Supposent-elles un changement de paradigme dans la gestion et l'organisation d'entreprises dans notre pays ? Ou ne sont-elles, peut-être, qu'une conséquence logique de la production flexible, de la marchandisation expansive du capitalisme tardif et un écho d'initiatives qui irradient d'autres centres névralgiques d'entreprises?

Mots clé: innovation; ethnographie corporative; aliénation; post-fordisme; intangibles; gestion des ressources humaines; facteur humaine; entreprise de base humaine; création/ destruction d'entreprises.

\section{Abstract. The rise of "human-based enterprises» in Spain: a new paradigm or an inevitable outcome?}

This paper presents a number of ethnographic case study of Spanish so-called "human-base enterprises", since they enhance the human factor as a competitive advantage. These companies do share certain traits: they operate in the knowledge economy, founders are talented young entrepreneurs, knowledge from humanities and arts are usually applied, are flexible and cooperative, apply horizontal management, and do not necessarily pursue profit as their main objective. These companies stand out in opposition to traditional businesses and management models, and human-centered innovation is stressed over classical technological and scientific innovation. However, are these enterprises genuinely groundbreaking? Do they lead a real paradigm shift in management and business organization in our country? Or, instead, are they a logical outcome of flexible production, commodification and the expanding character or late capitalism?

Keywords: innovation; business ethnography; alienation; post-Fordism; intangibles; human-based corporations; human factor; human resources management; business creation and destruction. 


\section{Sumario}

Introducción Discusión: paradojas y tensiones

Las empresas de base humana: de las empresas de base humana contexto e investigación Referencias bibliográficas

De la producción en masa a las empresas de base humana

\section{Introducción}

La correspondencia entre nivel de productividad y la calidad de las relaciones laborales es una cuestión que ha ocupado y preocupado a teóricos sociales clásicos en general (Karl Marx, Adam Smith y Max Weber, entre otros) y a sociólogos y antropólogos del trabajo en particular. Hace casi un siglo, entre 1924 y 1933, el estudio llevado a cabo en la planta Hawthorne de la empresa Western Electric (Chicago) por E. Mayo y sus asociados arrojó unos resultados sorprendentes: aunque el estudio estuvo inicialmente diseñado para medir la relación entre el nivel de iluminación en el lugar de trabajo y la productividad de los empleados, acabó poniendo de manifiesto el peso específico de los factores emocionales, psicológicos y sociales en el funcionamiento de las organizaciones, la importancia de las relaciones informales en el trabajo y, especialmente, que los trabajadores ponían más empeño en su trabajo si pensaban que sus jefes se interesaban por su bienestar. Estos estudios dieron lugar a los conceptos y políticas de los llamados recursos humanos, de desigual aplicación en el mundo empresarial, donde en general la dimensión humana se ha supeditado a los criterios de productividad, eficiencia e innovación tecnológica (Reygadas et al., 2012).

Recientemente parece haber aparecido una grieta en lo que parecía una asociación directa entre productividad e innovación tecnológica, con la emergencia de lo que aquí denominamos, con reservas y a falta de un término más adecuado o esclarecedor, empresas de base humana; empresas que, si bien no constituyen un grupo homogéneo, presentan características por las cuales el factor humano (en su vasta manifestación) se pone al frente y se convierte en motor, instrumento e incluso objetivo de la innovación.

Las cuestiones que se plantean aquí son: ¿qué procesos explican que el factor humano constituya ahora una novedad?, ¿nos hallamos realmente ante una innovación organizativa y empresarial?, ¿qué dilemas y contradicciones entraña este giro? Pensamos que la etnografía de los negocios y la antropología en general tienen algo relevante que decir a este respecto.

A partir de una serie de estudios de caso sobre empresas de base humana de reciente creación y de un marco teórico suficientemente amplio que nos permita ver en perspectiva la evolución del sector productivo e industrial, trataremos de ofrecer respuestas, siempre tentativas, a estas cuestiones. En lo subsiguiente, la primera parte contextualiza la investigación en la cual se basa 
este escrito. La segunda parte presenta una exposición de los diversos modelos de producción que nos conducen a este tipo de empresas. La tercera parte enumera las características de tales empresas de base humana. Y la última parte presenta una discusión a modo de conclusión.

\section{Las empresas de base humana: contexto e investigación}

Este análisis se basa en dos proyectos de investigación financiados en los que la antropología del empresariado y la etnografía de los negocios constituyen el eje común. ${ }^{1}$ En particular, los casos y la discusión aquí desarrollada parten de un proyecto de investigación pionero, promovido y subvencionado por la Escuela de Organización Industrial (EOI), por el cual se toma el pulso de la llamada nueva economía española. Este estudio fue inicialmente encargado a un equipo de expertos, antropólogos y etnógrafos procedentes de distintas universidades con el objetivo de estudiar aquellas empresas que introducían o aprovechaban saberes de las humanidades y las ciencias sociales. ${ }^{2}$ Se priorizó la aproximación etnográfica y las metodologías cualitativas usualmente asociadas. Tras la selección de las empresas según unos criterios de partida (ver más abajo), uno o varios etnógrafos realizaron una estancia de varios días (entre tres y cinco aproximadamente) en el lugar de trabajo de la empresa, para observar la realidad cotidiana, entrevistar en profundidad a distintos agentes (fundadores, propietarios, trabajadores, asociados, etc.) en contextos formales e informales y recabar información de archivo y administrativa, y a través de grupos de discusión o focales.

Pronto se evidenció, sin embargo, que la categoría genérica de empresa humanista englobaba un elenco más amplio y que lo humano, como factor, trascendía aquel etiquetaje disciplinario. Por ello se optó por la noción polisémica de empresas de base humana. La elección de estas empresas se rigió por una serie de criterios discutidos en el seno del equipo ${ }^{3}$ y se priorizaron aquellas en

1. «Empresas de Humanidades 20+20. Estudio de sectores Nueva Economía 20+20», Escuela de Organización Industrial (Ref. PS20121230). IP Francisco Cruces. «Perfiles del empresariado étnico en España. Una aproximación a las causas, dinámicas y espacios transnacionales de empresarios inmigrantes» (CSO2009-07057). Ministerio de Ciencia e Innovación (de 01/01/2010 a 31/12/2012). IP. José Luis Molina. Asimismo, esta publicación se ha beneficiado de las siguientes fuentes de financiación: una ayuda para acciones de cooperación en el marco de la Comunitat de Treball dels Pirineus AC065723 (2010 XI-CTP) de l'Agència de Gestió d'Ajuts Universitaris i de Recerca de la Generalitat de Catalunya; la Acción Complementaria (CS 02011-13651-E) del Ministerio de Ciencia e Innovación.

2. El artículo es deudor de la investigación realizada por este equipo: Luis Reygadas (UAM-I, México), Francisco Cruces (UNED), Humberto Matas e Irene Estrada (dnx|Designit), Nancy Konvalinka (UNED), Hugo Valenzuela (UAB), Diego Herranz (UNED), Sandra Fernández (UNED), Montserrat Cañedo (UNED) y Carlos Montes (UNED). Los resultados del proyecto han sido dispuestos por la EOI en acceso libre y pueden hallarse y descargarse en el siguiente enlace: http://www.eoi.es/mediateca/video/1470

3. Todos los estudios financiados por la EOI para el proyecto Empresas 20+20 se basan en una muestra de veinte empresas. En este caso, cuatro de esas veinte empresas fueron etnografiadas por uno de los autores de este artículo. 
las que: $a$ ) el factor humano es relevante dentro de la empresa (se privilegia el aspecto humano del funcionamiento de la organización: potencian la comunicación, horizontalidad en la gestión, participación, etc.); $b$ ) se potencia el factor humano desde la empresa hacia su entorno (se privilegia un trato diferencial a los actores de su cadena de valor en términos de atención, servicio postventa, procesos de cocreación, etc.); c) el factor humano es el objetivo o medio (se presta especial atención al capital social aprovechando el potencial de las redes sociales, o se incorpora la dimensión humana en el proceso de diseño, en las metodologías, en el espacio de trabajo, etc.); $d$ ) el factor humano es un recurso diferencial (por ejemplo, empresas étnicas o donde la diversidad y variabilidad social suponen una ventaja competitiva en términos de recursos), y $e$ ) el factor humano como capital humano (empresas que incorporan las humanidades o las ciencias sociales para generar riqueza).

\section{De la producción en masa a las empresas de base humana}

La productividad industrial ha ido generalmente asociada a la aplicación de innovaciones tecnológicas cada vez más eficientes, sofisticadas y racionales. Recientemente, sin embargo, la innovación está desplazando el foco de atención desde la tecnología hasta las personas, lo cual también conlleva una transformación de las características del proceso de producción y el tipo de consumidor. Ambos campos merecen una investigación en profundad en sí mismos, pero aquí lo relevante es notar su interrelación: el modo en que, en el actual régimen de acumulación, se retroalimentan producción y consumo (e. g. Aglietta, 1979).

Aunque autores pioneros indagaron antes en esa dirección, ${ }^{4}$ las tesis de Frederick Winslow Taylor suelen considerarse el inicio de la organización científica del trabajo cuyo objetivo era el incremento de la productividad mediante la fragmentación, la racionalización y la mecanización de los procesos industriales, por los cuales el operario quedaba reducido a un apéndice, un engranaje, de la máquina productiva. En The priciples of scientific management (1911), basados en el estudio empírico en la industria del acero, Taylor concluye que la productividad se incrementaba fragmentando las tareas y sometiendo el trabajo a rigurosos estándares de tiempo y espacio, así como estableciendo una remuneración en función de la productividad. Este sería el inicio de un proceso de progresiva alienación y descualificación del obrero, parejo a la creciente depreciación de la producción artesanal, y el despegue de la innovación tecnocientífica puesta al servicio del aumento de la productividad.

Pocos años más tarde, en 1914, Henry Ford introdujo su salario de 5 dólares por hora en una jornada de ocho horas en el contexto de las cadenas de montaje de automóviles de Dearborn, Michigan, lo que dio un origen simbólico al modelo fordista. Lo original de Ford, que potenciaba la ten-

4. Algunos referentes pueden rastrearse en Charles Babbage, Frank Bunker Gilbreth o incluso en Adam Smith y su An inquery into the nature and causes of the wealth of nations (1776). 
dencia tecnológica anterior, era su visión: entendió bien la relación virtuosa entre producción en cadena y consumo de masas. Este modelo, animado por el keynesianismo, enfatizaba el incremento de los salarios como motor de la demanda y reactivador de los mercados tras la profunda crisis del 29. El fordismo alumbra una nueva forma de vida, una cosmovisión, una estética y una psicología. En definitiva, tiene lugar el surgimiento de la sociedad de consumo, por la cual se produce una creciente mercantilización de la cultura con radicales cambios en los procesos de trabajo y en los hábitos de consumo (Harvey, 1991; Jameson, 1995; Marcuse, 1964). Este sistema se sostuvo gracias a la legión de obreros-consumistas y estuvo legitimado por la preponderancia financiera y militar de Estados Unidos y, por supuesto, por el triunfo de la expansión de la economía de mercado.

Sin embargo, a partir de los años setenta del pasado siglo, el fordismo norteamericano, y por inercia el del resto de los países ricos, comenzó a desplomarse debido al encadenamiento de complejos factores: su excesiva rigidez, la caída de la demanda interna, el embargo del petróleo por los países de la OPEP en 1973 y la creciente competencia que establecieron países de Asia y Europa con Estados Unidos. Japón, en el aislamiento que siguió a la Segunda Guerra Mundial, logró absorber, replicar y mejorar las técnicas de producción y originó el sistema toyotista, abanderado de la nueva era flexible que acabó imponiéndose en todos los países ricos. La expansión del sistema flexible coincide con el auge del capitalismo global, pero también con cierta hibridación empresarial por la cual conviven grandes corporaciones transnacionales (con gran integración horizontal en forma de clústeres empresariales que gravitan en torno a centros de investigación e institutos tecnológicos), con empresas nacionales que aplican una gran externalización de costes (subcontratas, franquicias, etc.), formas de fordismo periférico (talleres de explotación obrera), economías informales, viejos sistemas de trabajo doméstico y una progresiva presencia de empresas étnicas (Van Delft et al., 2000). En el ámbito social y político, esta fase coincide con la revolución neoliberal impulsada por Margaret Thatcher y Ronald Reagan en los años ochenta, en la cual el elevado desempleo estructural, el endeudamiento y la inflación se acompañaron de duras medidas de austeridad, recorte fiscal y rebajas en las ayudas sociales, lo que potenció, en consecuencia, el individualismo y la cultura emprendedora.

En la era flexible, «las economías extensivas han superado a las economías de escala» (Harvey, 1991: 155). La producción en cadena fordista se convierte en producción "paquetizada", "customizada" y dirigida a sectores de consumidores adiestrados, exigentes y selectivos que buscan bienes distintivos, posicionales. El gran almacenaje industrial propio de la antigua era industrial es sustituido por un aprovisionamiento justo a tiempo y mejor adaptado a las demandas cambiantes y caprichosas. Por flexibilidad, en este contexto, debe entenderse una doble acepción (Smith, 1997): una flexibilidad numérica que polariza el mercado laboral en un núcleo muy especializado y flexible (trabajadores de cuello blanco) y en una periferia temporal y precaria (con una creciente feminización y etnificación de la mano de obra); y una flexibilidad 
formal que revoluciona la organización del trabajo en muy diversos aspectos: la producción es altamente adaptable gracias a las tecnologías de la información y sistemas inteligentes, lo que facilita el trabajo móvil y desterritorializado (posibilidad de trabajar en espacios no necesariamente laborales). El contexto de la producción funcional y fragmentaria, propio del fordismo, se concentra y aglomera en clústeres donde se interconectan procesos con mayor eficiencia. La cultura de empresa refuerza el trabajo en equipo, la formación laboral continua, la rotación de tareas y el control de calidad en (y no al final de) el proceso, que se hace «más intensivo, rápido, [lo cual permite] bombear, fundamentalmente, trabajo del trabajador" (Kenney y Florida, 1993: 264). El salario del operario se expone a un sistema de bonificación por productividad, y se le exige a cambio de todo eso mayor responsabilidad, implicación y atención a los procesos productivos, lo cual intensifica el estrés en el puesto de trabajo.

Otra característica de esta fase productiva es la expansión, podríamos decir hipertrofia, del sector de los servicios. Pero las relaciones entre consumidores y trabajadores se hacen más mecánicas e impersonales - aunque sonrientes (cf. Velasco et al., 2009) — y la eficacia, el cálculo, la predicción y el control dominan una sociedad cada vez más macdonaldizada (Ritzer, 1996). No solo una parte del proceso productivo se transfiere al mismo consumidor ("hágalo usted mismo»), sino que el cliente efectúa un trabajo de supervisión sin saberlo - mediante el acceso visual al puesto de trabajo. Todo esto evidencia que, bajo el discurso participativo, yace en muchos casos un control más sutil y táctico del trabajador.

Como en la producción flexible de bienes, dirigida a sectores específicos de la sociedad, la producción y el diseño de servicios también se "paquetiza" y se orienta a diferentes sectores de la sociedad. Estos servicios comienzan a incursionar en nuevos territorios: el diseño de servicios centrado en las personas, la producción de eventos (espectáculos, etc.), el turismo experiencial, los servicios emocionales, etc. El ciclo parece cerrarse: desde el fordismo (producción en masa de bienes tangibles y consumo de masas) hasta la era flexible (mercantilización de intangibles centrados en un consumo individualizado). La sociedad moderna, democrática, populista y racionalizada va transformándose en una sociedad tardomoderna, individualista y fragmentada (Harvey, 1991 y Jameson, 1995). La transformación social queda claramente insinuada por la transición de la «macdonaldización» de la sociedad a su «disneylandización» (Bryman, 1995), dominada ahora por la temática (de restaurantes, centros comerciales, eventos...), la desdiferenciación (interconexión de las distintas áreas de consumo: alimentación, hostelería, ocio...), la promoción comercial y la creciente presencia del trabajo emocional (Paterson, 2006). La «desmaterialización» de las mercancías (Lee, 1993) coincide en los países ricos con la mercantilización de experiencias y sensaciones en detrimento de los bienes materiales, algo que algunos autores se han apresurado a etiquetar con el nombre de "economía de la experiencia» (Pine y Gilmore, 1999). En este contexto emerge con fuerza esa clase creativa (Florida, 2010).

La expansión de la economía de mercado en el sector servicios, como hemos visto, alcanza nuevas áreas de la vida: desde la producción cultural (Rowan, 
2010) hasta bienes centrados en las personas, pasando por todo tipo de ofertas de experiencias. Ahora bien, en un contexto productivo tan efímero y competitivo, marcado por la innovación y la constante adaptabilidad, la supremacía empresarial pasa a depender de la toma de decisiones rápidas y basadas en un conocimiento fiable. El control del conocimiento resulta crucial y estratégico para las empresas que incursionan en estos mercados.

En 1966 Peter Drucker publicó The effective executive y popularizó la noción de economía del conocimiento resaltando el papel de la imaginación o el conocimiento en la gestión empresarial. Si en el fordismo-taylorismo el obrero era un apéndice del proceso industrial, y en la producción flexible se requería su mayor implicación, en la economía del conocimiento el trabajador se sitúa en el centro del proceso, pues de este depende la innovación creativa. Para que la creatividad fluya, no obstante, es preciso alterar las condiciones del trabajo. La creatividad - apoyada en los portentosos desarrollos de las tecnologías de las comunicaciones y el transporte (Tapscott y Williams, 2006) y en el aprovechamiento de una inmensa alianza de trabajadores con elevado capital humano- pasa a constituirse como el nuevo baluarte de la innovación empresarial en la economía del conocimiento. Sin embargo, la creatividad no puede crearse, reproducirse ni replicarse por meros medios tecnocientíficos, porque requiere de facultades, procesos y capacidades cognitivas complejas y específicas. La creatividad (del latín creare, engendrar, tener hijos) se produce en conjunción con otros factores, como son la formación, el contexto social, los procesos conceptuales y lingüísticos o la motivación. Por si fuera poco, la creatividad posiblemente sea un proceso expuesto a un distinto grado de desarrollo según el contexto sociocultural del individuo. En el caso del moderno emprendedor schumpeteriano, se asocia a originalidad, autenticidad, adaptabilidad, excepcionalidad, independencia y pensamiento divergente - aunque en otros ámbitos sociales y culturales la creatividad no tiene por qué asociarse con esos criterios...

En definitiva, para que emane la creatividad se hace ahora preciso cuidar más el ambiente y el contexto, y esto incluye prestar mayor atención al espacio de trabajo, a las relaciones laborales, a la cultura de empresa o a los incentivos. Es aquí, en este preciso contexto, donde cabe explorar el florecimiento de las empresas de base humana.

La expresión empresas de base humana remite a un fenómeno variopinto, sincrético y transversal. Algunas empresas de este tipo poseen elementos propios de las empresas al uso, mientras que otras combinan aspectos hallados en otros tipos de economías - economías de la colaboración, etc. - o reflejan tendencias de las denominadas empresas postejecutivas (Jones, 2008). En general, este tipo de empresas englobaría a aquellas que cuidan particularmente las relaciones y el clima laboral, internamente y en el entorno de la red de valor (clientes, proveedores y usuarios); empresas que valoran y potencian el capital humano y social de sus trabajadores; que emplean en el proceso de producción y difusión conocimientos o procesos provenientes del campo de las ciencias sociales y las humanidades; o que integran la dimensión humana como elemento clave del diseño de procesos y productos. Pero lo novedoso no es la 
Tabla 1. Relación de las veinte empresas incluidas en la investigación

\begin{tabular}{|c|c|c|c|c|}
\hline $\begin{array}{r}\text { Empresa } \\
\text { (iniciales) }\end{array}$ & Sector & Campo & Localización & Tamaño \\
\hline $\mathrm{Cl}$ & $\begin{array}{l}\text { Economía del conocimiento. } \\
\text { Fusión de saberes }\end{array}$ & Nexo empresas, arte y humanidades & Bilbao & Micro \\
\hline DNX & $\begin{array}{l}\text { Economía del conocimiento. } \\
\text { Fusión de saberes }\end{array}$ & Consultoría, diseño e innovación & Madrid & Mediana \\
\hline FP & $\begin{array}{l}\text { Economía del conocimiento. } \\
\text { Fusión de saberes }\end{array}$ & Consultoría, diseño e innovación & Bilbao & Pequeña \\
\hline The $\mathrm{H}$ & $\begin{array}{l}\text { Economía del conocimiento. } \\
\text { Fusión de saberes }\end{array}$ & Innovación social & Madrid & Mediana \\
\hline Inv. & $\begin{array}{l}\text { Economía del conocimiento. } \\
\text { Fusión de saberes }\end{array}$ & Software libre & Pamplona & Pequeña \\
\hline Viz. & $\begin{array}{l}\text { Economía del conocimiento. } \\
\text { Fusión de saberes }\end{array}$ & Consultoría informática & Madrid & Mediana \\
\hline AA & Diseño y cultura & Arquitectura & Madrid & Micro \\
\hline CC60 & Diseño y cultura & Arquitectura & Madrid & Pequeña \\
\hline CdS & Diseño y cultura & Diseño & Valencia & Mediana \\
\hline$e-C$ & Diseño y cultura & $\begin{array}{l}\text { Intervención social y cultural. } \\
\text { Desarrollo local }\end{array}$ & Mérida & Pequeña \\
\hline PS & Diseño y cultura & Diseño & Madrid & Pequeña \\
\hline A Val. & Consumo alternativo & Producción alimentaria & Córdoba & Mediana \\
\hline Com. & Financiación alternativa & Financiación & Madrid & Pequeña \\
\hline Milh & Consumo alternativo & Producción alimentaria & Lugo & Pequeña \\
\hline Monv. & Consumo alternativo & Restauración & Barcelona & Mediana \\
\hline TM & Consumo alternativo & Distribución alimentaria & Madrid & Pequeña \\
\hline V & Financiación alternativa & Financiación de proyectos de arte & Barcelona & Pequeña \\
\hline $\mathrm{AC}$ & Cuidado y servicios personales & Turismo alternativo & León & Micro \\
\hline IMF & Cuidado y servicios personales & Reproducción asistida & Madrid & Mediana \\
\hline
\end{tabular}

Fuente: Reygadas et al., 2012: 31.

integración de esos aspectos, sino su combinación exitosa: la mayoría de estas empresas registran niveles de beneficio y crecimiento espectaculares.

La muestra analizada comprende veinte empresas, de las cuales cuatro fueron etnografiadas por el autor principal de este texto. Aunque los testimonios y las citas provienen de estas cuatro empresas, la descripción general se realiza basándose en las veinte empresas.

La inmensa mayoría de estas empresas son de reciente creación (menos de cinco años) y considerablemente pequeñas (con una media de empleados de diez personas, aproximadamente). El sector servicios en el que se ubican es diversificado y comprende el cuidado y servicios personales; programación de software libre; consultoría informática y sociocultural; consumo alternativo; arquitectura; la vinculación con empresas de artes y humanidades; financiamiento alternativo, micromecenaje o educación alternativa. El perfil 
del emprendedor responde predominantemente a un varón joven, de unos 35 años, proactivo, con una sólida aunque diversa formación superior (ingenieros, diseñadores, arquitectos, artistas...), sin previa experiencia empresarial $\mathrm{y}$, en muchos casos, con una dilatada experiencia vital, laboral o académica en el extranjero - por lo general en países del norte de Europa, Canadá o Estados Unidos. Estos emprendedores son individuos movidos por la búsqueda apasionada de experiencias, personas que anteponen recompensas intrínsecas (libertad, retos, reconocimiento o autorrealización) al beneficio económico y cuya actitud suele ser rupturista, iconoclasta o incluso anárquica - lo cual se refleja en la vestimenta, el lenguaje, las ideas o el propio nombre de la marca. Provocación sumada a talento, humildad y originalidad suele ser un cóctel habitualmente hallado en la base de estas empresas y en su proyecto empresarial - si lo tienen, pues otro rasgo característico es la ausencia de un proyecto empresarial formal y definido. Estas empresas no abandonan aspectos inherentes como la rentabilidad, la innovación tecnológica o la productividad, pero digamos que los supeditan a un factor humano que se halla en la forma de gestión y en la organización, en la estructura, en las relaciones laborales o en la estética y la marca. En definitiva, este grupo responde en gran medida al perfil de la «clase creativa» y, como tal, comparte la tres T: tecnología, talento y tolerancia, actitudes que no extrañamente suelen hallarse en los centros cosmopolitas (Sassen, 2000) donde se ubican las empresas analizadas (Valencia, País Vasco, Madrid, Barcelona), núcleos urbanos próximos a centros empresariales, industriales y de innovación tecnológica.

La organización suele alejarse de la verticalidad, la rigidez y la jerarquía. La gestión suele ser horizontal y se fomenta el trabajo cooperativo enfatizando el consenso, la comunicación - una comunicación que es constantemente alimentada y potenciada en el seno de la organización y entre los diferentes agentes de la cadena de valor. En el lugar de trabajo destaca un ambiente amigable, familiar, extrovertido, divertido y gregario. Como comentaba con ironía y ocurrencia uno de los fundadores de CdS: «CdS es un todo, es como Chacal, es un ente, puedes ver las acciones pero no a la persona. Si queremos un estudio polifacético no tiene sentido presentarlo bajo una o dos caras. Incluso las fotos hablan de colectivo.»

Todo esto suele ser, al menos en principio, coherentemente correspondido con una escasa dispersión de los niveles salariales entre los diferentes trabajadores y los gerentes; y existe un intento genuino por compatibilizar vida familiar y laboral y hacer del trabajo una actividad no necesariamente alienante. La sociabilización es un elemento crucial de este tipo de empresas y se potencia activamente (a menudo por individuos que poseen precisamente ese rol, el de dinamizador social de la empresa) incidiendo en la organización de eventos y actividades comunitarias — salir juntos, realizar fiestas, programar comidas, etc.

En estas empresas el espacio y el tiempo dejan de ser categorías acotadas o rígidas y devienen categorías maleables, dúctiles y, en definitiva, flexibles. Los espacios son transformables y el tiempo (horarios, jornadas, ritmos, etc.), elástico, lo que posibilita el trabajo en cualquier momento y tiempo gracias a las 
tecnologías disponibles. Los espacios se fusionan en un continuo: lo doméstico (una cocina) o lo lúdico (pufs gigantes esparcidos por el suelo para tumbarse) se entremezcla con lo profesional (una sala de reuniones, una sala de trabajo) y la dualidad ocio/trabajo disuelve sus límites constantemente: según un trabajador

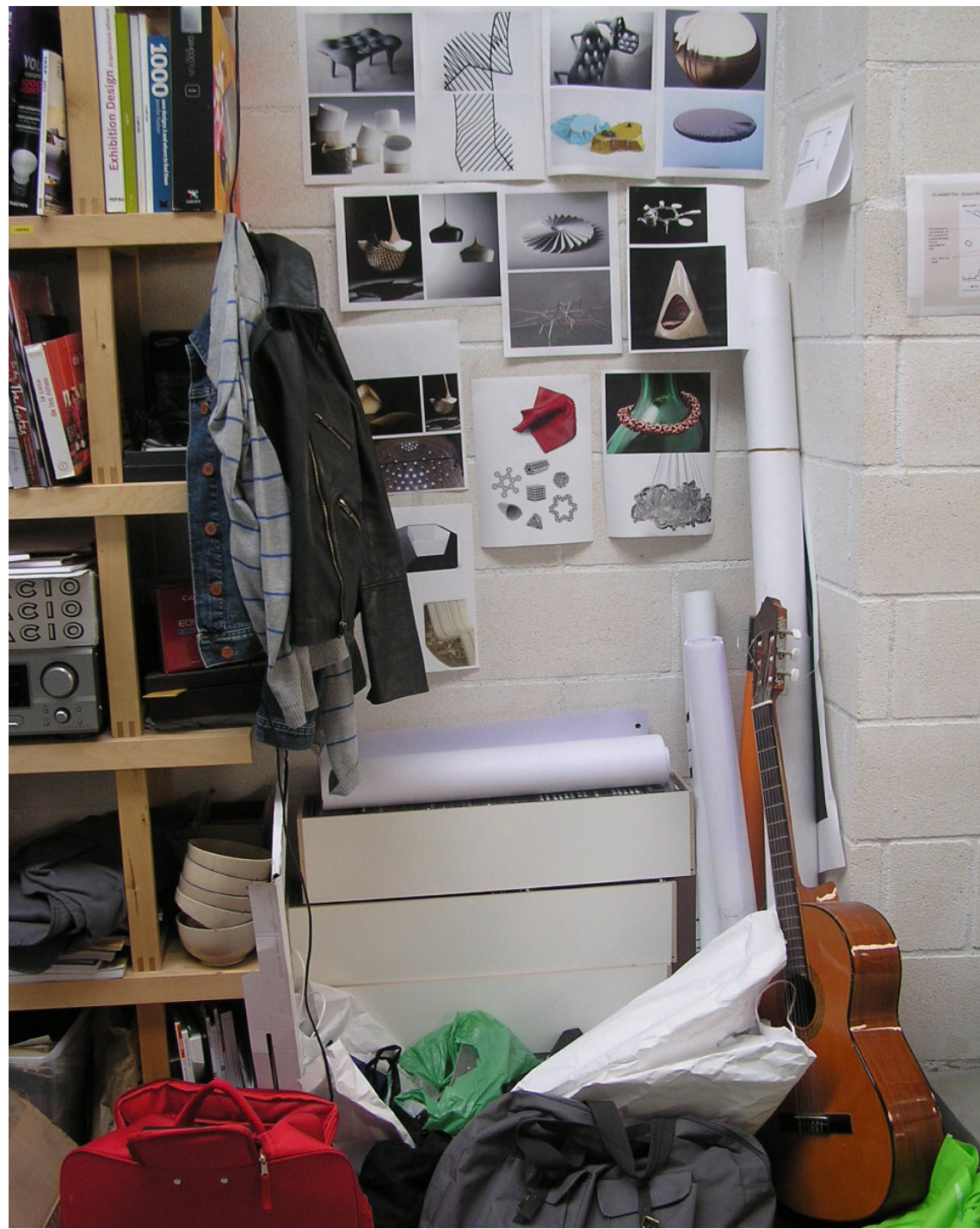

Ilustración 1. Un espacio lúdico en medio de un estudio de creación. Lo desarreglado, juvenil y extravagante (guitarra, estanterías utilitarias, aparato musical, mochilas...) se alterna con la creación y el diseño destinado a marcas de artículos de lujo (esbozos superiores). 
de la empresa $\mathrm{V}$, «lo podríamos hacer como hobby, es una actividad que nos gusta antes que una actividad laboral; es lo que nos engancha».

Espacios diáfanos, estética de diseño o incluso toques irreverentes, como colores chillones o excentricidades decorativas (véanse las fotografías), son la norma. En FP, por ejemplo, emplean mesas de un metro cuadrado con ruedas para posibilitar la movilidad, los encuentros o las reuniones breves entre los distintos trabajadores, de modo que los flujos entre personas, comunicaciones, procedimientos, materiales e ideas son constantes.

Los procesos y procedimientos suelen ser sincréticos, polifónicos y eclécti$\cos$ (se fusionan disciplinas, metodologías o incluso las voces de muy diversos agentes del proceso) y hasta cierto punto contraintuitivos, antiparadigmáticos, discordantes y divergentes. En una de las empresas existe un taller de anticreación en el que se destruyen objetos para evaluar alternativas procesuales o forzar «otras formas de hacer y pensar las cosas». Puesto que muchas de estas empresas profesan la innovación centrada en las personas (donde los cambios científicos y tecnológicos se acompañan de transformaciones sociales, culturales y organizacionales), no solo se modifica el producto, sino también el cómo, con quién y para qué se realiza. Eso implica centrarse en el mismo proceso y externalizar y subcontratar funciones y tareas que se estiman secundarias - aprovisionamiento, mantenimiento, administración, asesoría, limpieza, difusión, etc. Se potencia la negatividad de la sistematización de procedimientos en un intento de explotar al máximo las posibilidades creativas.

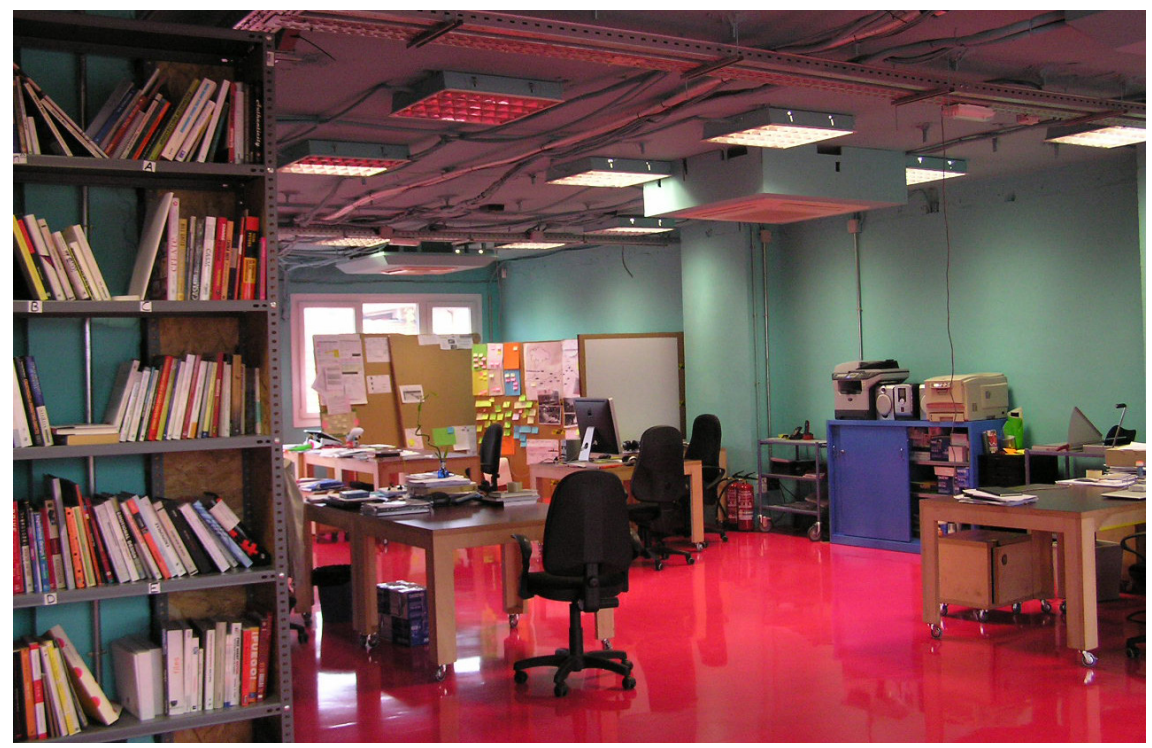

Ilustración 2. Espacio diáfano de la empresa FP: colores vivos, mesas móviles, arquitectura postindustrial, etc. Fuente propia. 
Los procesos de innovación y creación se suelen describir como algo divertido, lúdico y placentero: «Si no innovamos: nos vamos a divertir menos, la aportación que haremos a la sociedad es menor, y el crecimiento y desarrollo intelectual será pobre.»

En algunas empresas, no obstante, esta actitud no es una cándida o ingenua declaración de principios, sino un imperativo, una ética que se profesa y se fortalece desde el seno de la empresa - a veces de modo autoritativo, por el cual la creatividad deviene dogma. Esto abre las puertas a cierta estigmatización cuando se producen interferencias al proceso creativo: «Hay que evitar el mal rollo, a la gente que da mal rollo [...] todos tenemos un mal día, claro. Si alguien tiene un mal momento se le apoya, al día siguiente también, y se le ayuda hasta que esté bien. Pero si persiste y crea mal rollo, y lo transmite al resto, al final hay que despedir a esa persona.»

El aprendizaje continuo y el saber circulante resultan fundamentales. En efecto, en la creación de intangibles (ideas, procesos, eventos, servicios, experiencias...) el valor se incrementa con el uso y la circulación, al contrario de lo que suele ocurrir con los bienes de consumo tangibles, habituales. Por otra parte, existe una dignificación del saber artesanal — sin prescindir, paradójicamente, del uso de un inmenso elenco de dispositivos tecnológicos de última generación-y del valor creado colectivamente. "Este es el máster más importante que puedes hacer en tu vida", aseguraba una trabajadora de una de las empresas. El conocimiento es un valor distintivo y preciado y por ello

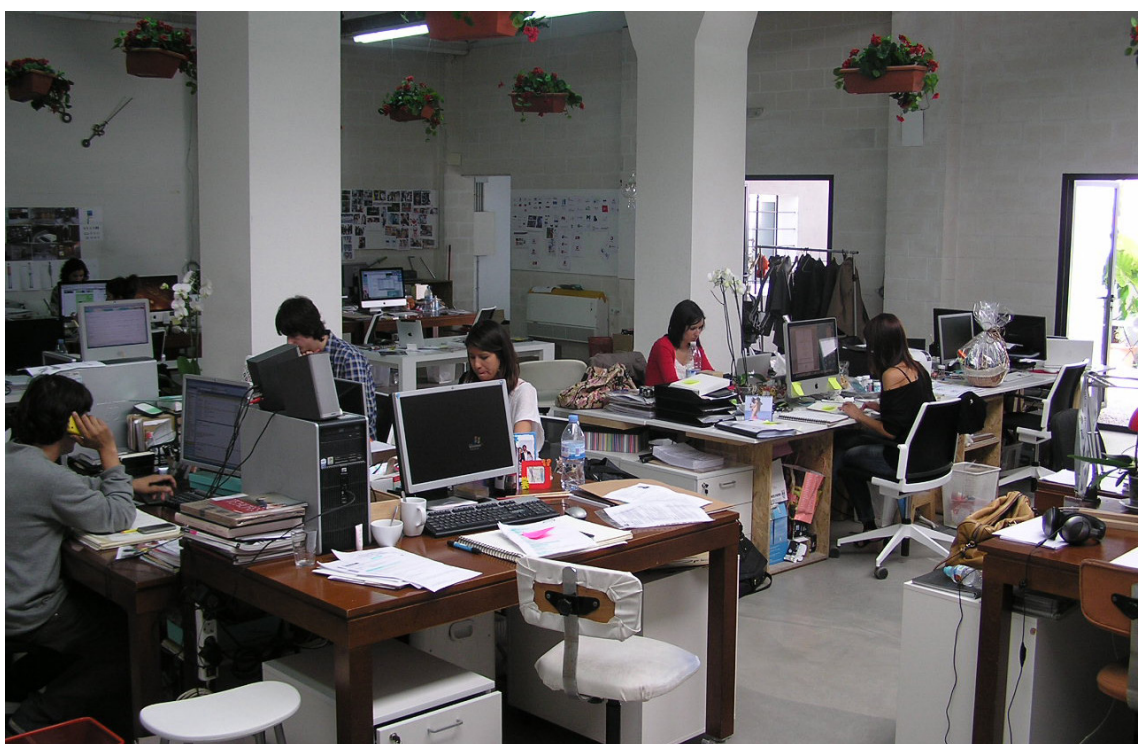

Ilustración 3. Espacio de trabajo de CdS. Ambiente gregario, amplio, fusionado y unificado; mobiliario móvil y maleable; interacción continua. Fuente propia. 
se promueve su creación, difusión y circulación dejando libertad al trabajador para que cree e innove sus propios proyectos personales en horario laboral - estrategia que han popularizado algunas grandes empresas de innovación tecnológica.

La tolerancia y la libertad son también actitudes que se incuban en este entorno. Durante una observación en una de esas empresas (CdS), un ingeniero se levantó, se dirigió a la cocina, hizo tortillas, preparó bocadillos y los distribuyó entre sus compañeros de trabajo. Mientras tanto, algunos trabajadores conversaban plácidamente en un fresco patio interior de la empresa mientras tomaban café y fumaban tranquilamente un cigarrillo. En otra empresa, un diseñador se levantó de su puesto de trabajo, se dirigió a una terraza interior y pintó a tiza, con traza, una puerta en una de las paredes. Eran actitudes habituales y nadie se inmutó, porque eso era lo que se pretendía (y se esperaba) cuando crearon algunas de estas empresas: «Cuando creamos CdS lo que nos apetecía era tener libertad, y lo que queríamos para nosotros lo queríamos para toda la gente que trabajara con nosotros.»

El reclutamiento de nuevos miembros no solo se basa en el talento, la experiencia o la formación, sino también en categorías subjetivas que resultan aquí muy relevantes: actitud, capacidad comunicativa, empatía, solidaridad, inteligencia emocional, etc. En algunas empresas estas actitudes extracurriculares son observadas y "evaluadas» para medir el grado de integración y encaje en la dinámica grupal mediante protocolos formales e informales, tales como juegos de rol, actividades en grupo o una simple comida con el resto de los empleados. "No queremos tiburones, preferimos empollones», afirmaba uno de los creadores de CdS. Tales rasgos personales, sin embargo, no son en absoluto triviales y, unidos a ambientes lúdicos, determinan el grado de elevadísimas ratios de productividad y creatividad. Como afirmó el creador de la empresa M: «Si tú eres feliz, tus clientes son felices, nosotros somos felices, todos somos felices». Por esa razón, la elección de los proyectos es selectiva, según el grado de desafío y los retos que propongan - «elegimos aquellos proyectos que nos hacen brillar los ojos", decía un entrevistado. Puesto que cada proyecto es un reto, el grado de implicación es muy elevado en el intento de imprimir una muesca de exclusividad, una esencia personal. Esto no solo refuerza la cultura corporativa, sino que también revaloriza el trabajo: cocrear, formar parte del proceso de producción, es lo que algunos autores identifican como el «efecto Ikea", que supone el incremento del valor de un objeto cuando el individuo se involucra en (y se identifica con) el proceso de producción (Norton, et al. 2011). Lo que se consigue con esto es, simplemente, que el proceso de trabajo sea menos alienante, en el sentido original que le dio Karl Marx al término. Además, existe una voluntad expresa por una cultura de empresa conciliadora: que posibilite la compatibilidad entre vida personal y laboral, que lubrique las buenas relaciones sociales en el interior, etc., y que se esmere por reproducir, fortalecer y expandir la esencia de la marca.

La cultura de empresa roza la utopía en algunos casos, si bien es sorprendente el grado de aparente humildad y autenticidad que rebosan algunos de 
los portavoces. El mantra «existen otros modos de hacer las cosas» es realmente efectista y, vinculado a compromisos sociales (ecologismo, sostenibilidad, reciprocidad, ética, humanidad...), refuerza la cultura empresarial y contagia a los actores implicados. Vocación, esfuerzo, pasión, cooperativismo, reciprocidad, humildad, transparencia, franqueza, búsqueda de sentido, diversión... forman un manto de valores, una superestructura, que realmente fortalece la cultura corporativa.

Muchas de estas empresas serían difícilmente imaginables sin tener en cuenta las tecnologías disponibles y la centralidad del capital y las redes sociales, y estas se potencian, se alimentan, se reproducen y se explotan por todos los medios posibles: en algunos casos son medios y en otros constituyen directamente fines.

\section{Discusión: paradojas y tensiones de las empresas de base humana}

Llegados a este punto, trataremos de ofrecer algunas respuestas tentativas a las cuestiones iniciales: ¿qué factores explican que el factor humano constituya ahora un activo tan relevante? ¿Nos hallamos ante una ruptura de paradigma, una innovación? ¿Qué dilemas y contradicciones entraña este giro? Asimismo mostraremos algunas de las tensiones más evidentes que se derivan de este tipo de empresa.

Buena parte de los países occidentales y ricos han transitado una misma progresión histórica desde una economía de base agropecuaria hasta llegar a una economía del conocimiento donde el principal producto deja de ser un bien tangible y deviene un servicio intangible, pasando por una economía industrial de producción en cadena y por una economía flexible y adaptada a sectores concretos de consumidores. Son los ritmos que ha establecido el capitalismo mundial y, sin negar una amplia variabilidad regional, son el patrón general. En esta progresión se percibe una intensificación de la productividad gracias al incremento del dato tecnológico y una gradual expansión de la economía del mercado y la biopolítica capitalista (Wolf-Meyer, 2011) a todas las esferas de la vida: el cuerpo y sus partes (Sharp, 2010), la cultura (Jameson, Rowan, 2010), la etnicidad (Comaroff y Comaroff, 2009), la experiencia (Bryman, 2004) y el ADN (Resnik, 2001). En este sentido, las empresas de base humana serían una derivación lógica, antes que una ruptura, de ese proceso de expansión económica mercantilista. Bajo esta perspectiva continuista, resulta que el grado de originalidad de la mayoría de estas empresas es solo relativo: algunos de los rasgos de su cultura corporativa recuerdan a empresas pioneras internacionales (IDEO, ZIBA, BMW, Apple, Google, Whole Foods, Microsoft, Starbucks...). Tapscott y Williams, autores de Wikinomics, señalan cuatro claves de éxito empresarial que no resulta difícil reconocer en las empresas analizadas: obertura, cooperativismo, reciprocidad y acción global (2006: 1). Sin embargo, no podemos subestimar el efecto de la glocalización, por el cual los factores globales originan fenómenos imprevisibles en el contexto local; un término que, irónicamente, tiene su origen en el marketing empresarial japonés 
(Khondker, 2004). Quizás en ese realojamiento de lo global en el contexto local es donde radica el efectismo de estas empresas.

Los más optimistas ven en las empresas de base humana una forma de imbricación inversa, por la cual lo social y lo económico - esferas que han tendido a separarse progresivamente hasta llegar a la sociedad de mercado (Polanyi, 1944; Dumont, 1982; Jameson, 1991; Molina y Valenzuela, 2006) — experimentan una suerte de reconciliación y vienen a conformar una misma esfera o dimensión, como aparentemente ocurre en ciertas sociedades simples (e. g., Malinowski, 1976). Sin embargo, parece que si hemos llegado a este punto es por la confluencia de dos fuerzas opuestas. Por una parte, por supuesto, en esta tendencia económica se percibe la influencia de un discurso y una praxis social alternativa, contracultural, utópica, que profesa que «hay otra manera de hacer las cosas» o que la economía - sobre todo en el momento de sofocante crisis que vivimos - requiere ser repensada y reformulada para que logre reconectar con necesidades y demandas sociales más genuinas e inmediatas. Por otra parte, sin embargo, la idea de sociabilizar la economía ya ha devenido, inevitablemente, un reclamo comercial apetecible y fructífero, como ha ocurrido con otras tantas categorías similares. ${ }^{5}$ Nunca podremos saber con certeza si bajo el discurso de la empresa de base humana yace una estrategia perversa o una voluntad genuina, pero, como en la eficacia simbólica (Lévi-Strauss, 1949) o en todo efecto placebo, es evidente que lo que hacen y profesan les funciona, y les funciona bien, y logran impregnar su talante a otros sectores empresariales: reconsiderar el bienestar en el trabajo, ponerlo de relieve por delante de otros aspectos empresariales (beneficio, rentabilidad, productividad, racionalidad técnica...) y convertirlo en ventaja competitiva - algo que el activista británico Robert Owen ya planteó en el siglo XVIII.

Hace una semana, mientras conducía camino a casa, una radio pública recibía con optimismo una idea «innovadora» procedente de una empresa de recursos humanos: proponían realizar entrevistas de trabajo mientras el candidato y el entrevistador tomaban café en un ambiente distendido. Una candidata entusiasta afirmaba lo siguiente: «Se trata de una idea muy bonita, muy humana». Cuando lo humano y lo social, como categorías emergentes en este nuevo viraje económico, están en boca de todos, quizás es momento de comenzar a replantearse un análisis de este movimiento como algo más sistémico, no coyuntural, que requiere un análisis más crítico y profundo.

Escribía David Harvey — hace ya más de dos décadas y haciéndose eco de los teóricos de la regulación (Aglietta, 1979; Lipietz, 1986 y Boyer 1986, citados en Harvey, 1991: 122) — que el sistema capitalista, para ser viable, debía salvar dos escollos: el primero deriva de la aparente anarquía por la cual se fijan los precios en el mercado (participan a partes desiguales el Estado, la mano invisible, los monopolios, las modas, etc.); el segundo se refiere a la capacidad

5. Véanse etiquetas como consumo sostenible, ecología responsable, turismo gay o banca ética (Hawthorne, 2012). Al fin y al cabo, nos encontramos frente a nuevos nichos con nuevas tipologías de consumidores, que suponen nuevas oportunidades de negocio. 
de ejercer suficiente control sobre la fuerza de trabajo para seguir garantizando la extracción de la plusvalía y ese control suele ejercerse mediante complejos y difusos mecanismos: habituación, coacción, sociabilización, educación, movilización de sentimientos sociales (ética del trabajo, lealtad, orgullo...), propensión psicológica (autorrealización, autonomía, eficacia...), etc.

Las empresas de base humana son un prodigio en ambos sentidos: en primer lugar porque logran colocar en el mercado un producto cuyo precio es totalmente subjetivo (experiencias, diseños personales, etc.). En segundo lugar, porque en estas empresas la productividad ha alcanzado altas cuotas, precisamente por no ejercer un control directo sobre el trabajador sino un control informal de todas las dimensiones de la vida personal. Como afirmó uno de los socios de CdS, «nadie es más exigente que uno mismo». Un trabajo vocacional, pasional, realizado en un ambiente amigable, con plena libertad creativa, donde se fomenta el reconocimiento, la reciprocidad y la colaboración y donde se da plena identificación con la marca y la cultura de empresa es, en definitiva, un medio que acelera la creatividad y potencia la productividad hasta niveles sorprendentes. En definitiva, el trabajo se percibe como menos alienante, no hay duda, pero el costo puede ser elevado. Es menos alienante porque los trabajadores se implican en el proceso creativo, no quedan separados de la relación social con sus compañeros de trabajo y se aprovecha su potencial humano; pero no siempre son propietarios de ese producto. La mercantilización de lo creado deja suculentos remanentes que no siempre recaen en el trabajador - luego la plusvalía extraída es elevada en algunos casos. La absorción por el proceso de trabajo, la ausencia de horarios, los plazos ajustados, la constante mezcla de vida laboral y doméstica, la flexibilidad del tiempo y el espacio también tienen consecuencias indeseables: autoexplotación, estrés, adicción al trabajo, perfeccionismo paralizante y problemas de conciliación con la vida familiar, que a su vez redundan en otros problemas psicológicos y sociales (Schieman y Young, 2010).

Como hemos descrito antes, frente a otras formas de organización de la empresa tradicional, la innovación en las empresas de base humana se caracteriza por la obertura, la reciprocidad y la circulación de los saberes y metodologías. Esto es así porque el valor del producto (generalmente intangible) se incrementa con su circulación y uso, al contrario de lo que suele ocurrir con los bienes de consumo tangibles y habituales - exceptuando categorías especiales como los bienes de lujo, coleccionables, objetos rituales, simbólicos, etc. Esta inversión de las categorías de uso y valor, en el ámbito de la mercantilización de intangibles (experiencias, eventos, cultura, procesos...), abre un dilema frente a las concepciones clásicas de propiedad privada, precio, valor de uso y valor de cambio. Pero la desintegración de la categoría de propiedad privada en este contexto podría ser también aparente. Primero, porque no fluyen todos, ni con la misma intensidad, los saberes o procesos implicados: las patentes y los protocolos creativos singulares se guardan celosamente - revelar el contenido de esos conocimientos fue causa de despido de un trabajador en una de esas empresas. Segundo, porque mientras que la paradoja es evidente en el proceso 
de producción, no parece darse en el proceso de consumo. Aunque el objetivo fundamental de estas empresas no es el lucro, el perfil de los consumidores responde a empresas de alto valor añadido (generalmente grandes corporaciones internacionales que buscan los servicios de creativos frescos) o consumidores con elevados ingresos o gran capital cultural. Esta paradoja vuelve a surgir a otro nivel: el personal de estas empresas pertenece a un club selecto en el marco del mercado de trabajo (dadas sus credenciales académicas y su entorno socioeconómico) cada vez más distanciado de una mayoría laboral crecientemente precarizada y empobrecida.

Algunas empresas de base humana reclaman una innovación centrada en las personas y no necesariamente tecnológica. Pero aquí cabe señalar dos contradicciones: la emergencia y la operatividad de estas empresas sería difícilmente imaginable sin el uso intensivo y extensivo de tecnologías de las comunicaciones y, de hecho, los espacios y procedimientos de trabajo están inundados de esta tecnología. Por otra parte, hay pocas creaciones tan humanas como la propia tecnología: cuando se habla de base humana, por lo tanto, se están seleccionando y privilegiando unas facetas determinadas de la naturaleza humana (idealizada, "paquetizada", comercializable) y no otras.

La relativa juventud de estas empresas no posibilita realizar una evaluación longitudinal ni una previsión a medio plazo. Algunas de ellas, en expansión, comenzaban a quejarse de ciertas dificultades propias para compatibilizar los objetivos del negocio y los objetivos humanos (la vida familiar y personal); y se atisbaban conflictos cuando en el seno de la empresa se daban crecientes desigualdades entre simples trabajadores y dueños, socios o accionistas —en términos de incentivos o salarios. Por la misma razón (la relativa novedad), apenas es posible conjeturar modos en los que podría operar la explotación y la resistencia (León Medina, 2009), pero en cualquier caso resulta obvio que estos mecanismos son más sutiles y difusos porque operan en entornos donde lo profesional se funde con lo emocional y lo subjetivo.

Una mirada amable de estas nuevas organizaciones laborales nos obliga a abrazar, con esperanza, cierta utopía: es innegable que las condiciones laborales resultan más gratas que en otros tiempos y contextos. Además, la constante sospecha crítica resulta inmovilizadora: ¿acaso es posible el cambio si no partimos de la idea de que es posible el cambio? Sin embargo, la expansión del capitalismo no responde, precisamente, a una fábula de liberación y altruismo: su evolución dicta una creciente sofisticación de mecanismos de control y opresión y —si seguimos a algunos teóricos de los estudios críticos de la gestión (Knights y Willmott, 1989; Willmott, 1993; Fernández Rodríguez 2007)— podríamos aventurar una o dos hipótesis menos clementes.

La aparente superación de antiguas formas de alienación, de control jerárquico y de formas rutinarias de poder en el contexto laboral también abren el escenario a la quintaesencia del control táctico: ¿qué hay más efectivo que el autocontrol y la autoexigencia en el proceso (creativo) de trabajo en un contexto en el que el control se desliza desde los agentes externos (el patrón, el grupo de trabajo, la organización empresarial o los valores sociales del momento...) 
hasta el sujeto? Este panóptico supremo (el yo controlado y explotado por el yo) deriva de una forma de dictadura de la creatividad por la cual el sujeto se ve presionado a extraer constantemente lo mejor de si mismo. Este tipo de explotación, podríamos afirmar, es el correlato lógico de la sociedad individualista y, sin embargo, no deja de abrir la puerta, como bien nota David Graeber (2012), a una última y gran paradoja: buena parte de las empresas postejecutivas de vanguardia más exitosas de nuestra era de capitalismo avanzado responden a principios no de rampante neoliberalismo sino de comunismo - horizontalidad, cooperación, «a cada cual según sus necesidades, cada uno según sus posibilidades».

\section{Referencias bibliográficas}

AgLIETTA, Michel (1979). Regulación y crisis del capitalismo: la experiencia de los Estados Unidos. Madrid: Siglo XXI.

BRYMAn, Allan (2004). The Disneyization of Society. Londres: Sage Publications.

Comaroff, John L. y Comaroff, Jean (2009). Ethnicity, Inc. Chicago: University of Chicago Press.

DuMONT, Louis (1982). Homo aqualis. Madrid: Taurus.

FERNÁNDEZ RodríGueZ, Carlos Jesús (ed.) (2007). Vigilar y organizar. Una introducción a los Critical Management Studies. Madrid: Siglo XXI.

FLORIDA, Richard (2010). La clase creativa. La transformación de la cultura del trabajo y el ocio en el siglo XXI. Barcelona: Paidós.

- (2002). The rise of the creative class (and how it's transforming work, leisure, community and everyday life). Nueva York: Basic Books.

Graeber, David (2012). En deuda. Una historia alternativa de la economía. Madrid: Ariel.

HARRIS, Marvin (2000 [1984]). La cultura norteamericana contemporánea. Una visión antropológica. Madrid: Alianza.

HAWTHORNE, Fran (2012). Ethical chic: The inside story of the companies we think we love. Boston: Beacon Press.

HARVEY, David (1991). The condition of postmodernity. An enquiry into the origins of cultural change. Cambridge, MA y Oxford: Blackwell.

JAMESON, Fredric (1991). Postmodernism, or the cultural logic of late capitalism. Durham, NC: Duke University Press.

JONES, Andrew (2008). The Innovation Acid Test: Growth through design and differentiation. Londres: Triarchy Press, Ltd.

KHONDKER, Habibul H. (2004). "Glocalization as globalization: evolution of a sociological concept». Bangladesh e-Journal of Sociology, 1 (2).

KNighTS, David y WillmotT, Hugh (1989). «Power and subjectivity at work: from degradation to subjugation in social relations». Sociology, 23 (4): 535-58.

LEE, Martyn (1993). Consumer culture reborn. The cultural politics of consumption. Londres: Routledge.

LEÓn Medina, Francisco (2009). «La lógica de los trabajadores. Un estudio sobre la racionalidad, la autonomía y la coherencia de las prácticas y los significados de los trabajadores». Revista Internacional de Sociologia, 67 (1): 135-60.

LÉVI-STRAuss, Claude (1949). «L'efficacité symbolique». En: (1958) Anthropologie structurale. París: Plon. P. 205-26. 
MarCuse, Herbert (1964). One-dimensional man. Studies in the ideology of advanced industrial society. Londres: Routledge \& Kegan Paul.

MALINOWSKI, Bronislaw (1976). "La economía primitiva de los isleños de Trobriand». En: Godelier, Maurice (comp.) (1976). Antropología y economía. Barcelona: Anagrama. P. 87-101.

Molina, José Luis y Valenzuela García, Hugo (2006). Invitación a la antropología económica. Barcelona: Bellaterra.

Norton, Michael I.; Mochon, Daniel y Ariely, Dan (2011). «The IKEA effect. When labor leads to love». Papeles de trabajo. Harvard Business School.

PAterson, Mark (2006). Consumption and everyday life. Londres y Nueva York: Routledge. The New Sociology Series.

PIne, B. Joseph y GILmORE, James H. (1999). The experience economy: work is theatre and every business a stage. Harvard: Harvard Business School Press.

POLANYI, Karl (1944). The great transformation. The political and economic origins of our time. Boston: Beacon Press.

RESNIK, David B. (2001). "DNA patents and human dignity». The Journal of Law, Medicine \& Ethics, 29 (1): 152-65.

REYGADAS, Luis et al. (2012). Sectores de la nueva economía 20+20. Empresas de Humanidades. Madrid: Fundación EOI.

RITZER, George (1993). The McDonaldization of society: An investigation into the changing character of contemporary social life. Thousand Oaks, CA: Pine Forge Press.

Ross, Andrew (2001). "No-collar labour in America's "new economy”". Socialist Register, 37: 77-87.

Rowan, Jaron (2010). Emprendizajes en cultura. Discursos, instituciones y contradicciones de la empresarialidad cultural. Madrid: Traficantes de Sueños.

SASSEN, Saskia (2000). Cities in a world economy. Thousand Oaks, CA: Pine Forge Press.

Schieman, Scott y Young, Marisa (2010). "The demands of creative work. Implications for stress in the work-family interface». Social Science Research, 39: 246-59.

SHARP, Lesley A. (2000). "The commodification of the body and its parts». Annual Review of Anthropology, 29: 287-28.

SMITH, Vicki (1997). "New forms of work organization». Annual Review of Sociology, 23: 315-39.

TAPSCOTT, Don y Anthony D. Williams (2006). Wikinomics. How mass collaboration changes everything. Nueva York: Penguin Group.

VAlEnZUEla García, Hugo (2007). «Una indagación antropológica sobre el trabajo y las profesiones». En: Blanco, Xavier y Mejri, Salah (eds.) (2007). Los nombres de profesiones. Enfoques lingüísticos, contrastivos y aplicados. Bellaterra: Servei de Publicacions de la Universitat Autònoma de Barcelona. P. 215-28.

VAn Delft, Hans; GorTer, Cees y Nijkamp, Peter (2000). «In search of ethnic entrepreneurship opportunities in the city: A comparative policy study». Environment and Planning C: Government and Policy, 18 (4): 429-51.

Velasco Maíllo, Honorio M. et al. (2009). La sonrisa de la institución. Confianza y riesgo en sistemas expertos. Madrid: Editorial Universitaria Ramón Areces.

WillmotT, Hugh (1993). «Breaking the paradigm mentality». Organization Studies, 14: 681.

Wolf-Meyer, Matthew (2011). «Natural hegemonies: Sleep and the rhythms of American capitalism». Current Anthropology, 52 (6): 876-95. 